\title{
Nanoelectronic Devices Based on Carbon Nanotubes
}

\author{
Victor Dmitriev ${ }^{1}$ Fernando Gomes ${ }^{1}$, Clerisson Nascimento ${ }^{1}$
}

\begin{abstract}
Carbon nanotubes are possible building blocks in the development of new generation of electronic devices. The carbon nanotubes allow one fabrication of devices using nanometric scales. They can be used in projects of a wide range of electronic and optoelectronic components such as diodes, transistors and interconnection elements, among others. This paper presents the state-of-the-art of this area, emphasizing the influence of quantum effects on the characteristics of such components.
\end{abstract}

KEYWORDS: Nanoelectronics, Carbon nanotubes, Nanotransistors.

\section{INTRODUCTION}

Carbon nanotubes (CNT) with different configurations are the most studied nanomaterials since their discovery in 1991 by S. Iijima (1991). They present a single hollow cylinder with one or several layers of carbon atoms. The CNTs behave as one-dimensional (1-D) systems since their diameters are on the nanometric scale and are much smaller as compared to their lengths. 1-D nature of CNT defines their special physical properties, which have been used in design of novel electronic devices. It is expected that the nanocomponents based on CNT will have some of electrical and optical parameters far superior than the existing microelectronic components based on $\mathrm{Si}$, $\mathrm{Ge}$ or GaAs semiconductors (Dragoman and Dragoman, 2006).

There is a relentless pursuit of getting smaller and smaller devices with low energy consumption and high processing speed in many areas, including aerospace technology. In this sense, single-wall carbon nanotubes (SWCNT) have been very promising because, depending on their chirality and diameter, they may exhibit metal or semiconducting behavior (Yao et al., 1999). This allows one to project good conducting wires and p-n junction in nanometric scale.

In this paper, we present a review of electrical properties of some components based on SWCNT. In particular, interconnecting elements, Schottky diodes and field-effect transistors are discussed. We make a comparison of the nanoelectronic components based on $\mathrm{CNT}$ and the corresponding microelectronic devices and discuss the principal problems in their modelling. 


\section{NANOELECTRONIC COMPONENTS BASED ON CNT CNT AS INTERCONNECTION ELEMENTS}

One of the first ideas for applications of SWCNT with metallic characteristics is the use of them as interconnectors in integrated circuits. A great advantage of carbon nanotubes is a possibility of ballistic transport over long length. The regime of ballistic transport, in contrast to diffusive one, is characterized by absence of scattering or collision of moving electrons in the CNT structure (Wong and Akinwande, 2011).

Integrated circuits with a large-scale integration can have billions of components. Before CNT can be used as interconnection wires, there are some challenges that must be overcome. All tracks that connect internal components of the integrated circuits suffer some unwanted phenomena such as delays, heat dissipation, noise, jitter and electromigration. The metallic CNT can serve as an alternative to overcome these challenges. They are characterized by a relatively long mean free path, high current density and resistance to electromigration (Wong and Akinwande, 2011; Javey and Kong, 2010).

Below, we describe the interconnection elements based on metallic SWCNT as a possible replacement of copper tracks. Due to 1-D nature of SWCNT, for a given applied external electric field, only a small number of channels or sub-bands are involved in the process of charge transport. The finite number of states means discretization of these states' energy levels. This leads to some quantum effects that are described by a quantum resistance, quantum capacitance and kinetic inductance, which are very different from the corresponding classical concepts of resistance, capacitance and inductance (Wong and Akinwande, 2011).

\section{Quantum Resistance}

Ideally, the good quality interconnection resistance must be negligible when compared to the resistance of the devices to be interconnected. The resistance that appears at the interface between a metal contact and a metallic SWCNT is called the quantum resistance. In a metallic SWCNT at low energies, i.e. energy of the order of a few electron volts, only two 1-D sub-bands are involved in the electrical transport. If the transportation is ballistic, the value of the quantum resistance at the interface between the metal contacts and the metallic SWCNT is $R_{q}=6.5 \mathrm{~K} \Omega$ (Wong and Akinwande, 2011; Javey and Kong, 2010).

\section{Quantum Capacitance}

As far as the quantum capacitance's effect is concerned, it appears also only in systems with finite number of states (Wong and Akinwande, 2011; John et al., 2004). This quantum capacitance $\left(C_{q}\right)$ is defined by the additional energy required to release charge carriers in systems due to quantization of the energy levels of quantum states. This capacitance is only noticeable in systems with a very low density of states (DOS). The value of the $C_{q}$ is much smaller than the value of the classical (geometric) electrostatic capacitance $\left(C_{e s}\right)$. As the $C_{q}$ is in series with the $C_{e s}$, the result of this combination is the dominance of the $C_{q}$. However, in structures with a large number of free electrons, the $C_{q}$ is very large when compared with the $C_{e s}$. As a result, there is a predominance of the $C_{e s}$. The value of $C_{q}$ of metallic CNT is around of $310 \mathrm{aF} \mu \mathrm{m}^{-1}$ (Wong and Akinwande, 2011).

\section{Kinetic Inductance}

The classical magnetic inductance $\left(L_{m}\right)$ is associated with the magnetic field and the corresponding magnetic energy due to charges motion. The kinetic inductance $\left(L_{k}\right)$ is associated with the kinetic energy of the electrons in motion. In case of ballistic transport in the CNT, the kinetic inductance is relatively large since the kinetic energy is not dissipated due to scattering. The $L_{k}$ is in series with the classical $L_{m}$. Due to the relatively long mean free path, which is characteristic of the CNT, the $L_{k}$ for signal frequencies above $100 \mathrm{GHz}$ becomes appreciable, which is of great importance in high-frequency electronic applications. The $L_{k}$ value for metallic CNT is about 3,2 $\eta H_{\mu m}^{-1}$ (Wong and Akinwande, 2011). This value takes into account the degeneracy of spin.

Degeneracy occurs when there are two or more quantum states with the same energy. The Pauli exclusion principle states that two electrons can not be in the same state. So, in order to accommodate two electrons in one state, the spins of the electrons have to be different, i.e. one electron with spin up and another electron with spin down. This is degeneracy of spin.

\section{Transmission Line Model of CNT}

A model of metallic CNT as a transmission line with distributed elements is presented in Fig. 1 . In this equivalent scheme, $C_{q}$ and $L_{k}$ effects were taken into account (Wong and Akinwande, 2011). In Fig. $1, R_{c} / 2$ is the input and output contact resistance of line, i.e. we deals with two equal metal contacts, and the $R_{c}$ must be divided into two. $R$ is the resistance per unit length of the metallic CNT, excluding $R_{c}$ This term describes ohmic losses in diffusive regime. $\Delta x$ represents the incremental length of line and $\Delta \mathrm{L}$ is the length of the transmission line.

The transmission lines are characterized by the characteristic impedance $\left(Z_{0}\right)$ and the propagation constant $\gamma$ (Sadiku, 2004). 
The propagation constant is in general a complex number. The real part $(\alpha)$ and the imaginary part $(\beta)$ represent the attenuation and phase constant of the transmission line, respectively. With the given parameters of the transmission line in Fig. $1, Z_{0}$ and $\gamma$ can be determined using the classical transmission line theory.

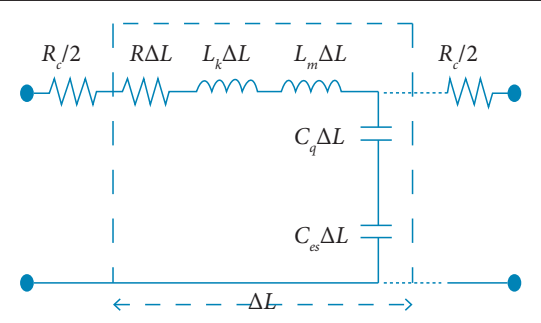

Figure 1. Transmission line model of CNT with distributed elements.

The transmission line can be considered lossy when the signal frequency is much below the critical frequency and lossless when the signal frequency is much above the critical frequency. This critical frequency is usually called zero frequency $\left(\omega_{z}\right)$ and it is defined as the ratio between $R$ and $L_{k}$ of the transmission line, i.e. $\omega_{z}=R / L_{k}$ (Wong and Akinwande, 2011). For a highquality SWCNT at room temperature and with the effective mean free path of $1 \mu \mathrm{m}, \omega_{z} \approx 318 \mathrm{GHz}$ (Wong and Akinwande, 2011).

In the lossless (ballistic) transmission regime, the propagation constant $\gamma$ of the line is a pure imaginary number which is proportional to the frequency of the signal, i.e. $\gamma=j \beta=j g \omega \sqrt{L_{k} C_{q}}$. In this equation, $g$ is a parameter that quantifies the electron-electron interaction, $j$ means that the variable is a complex number and $\omega$ is the angular frequency. When $g \sim 1$, the interaction is weak; and when $g \sim 0$, the interaction is strong (Wong and Akinwande, 2011). The line's $Z_{0}$, which, in this case, is given by $Z_{0}=1 / g \sqrt{L_{k} C_{q}}$, is a pure real number. It does not depend on the frequency. This means that a signal that propagates along this line does not suffer attenuation; besides, the voltage and the current of the line are in phase. The group velocity $v_{g}=\vartheta \omega / \vartheta \beta$ is constant, i.e. all the frequency components of the signal propagate along the line with the same velocity $v_{g}$ given by $v_{g}=v_{F} / g$, where is the Fermi velocity: $v_{F} \approx 10^{8} \mathrm{~cm} \cdot \mathrm{s}^{-1}$ (Wong and Akinwande, 2011).

In a lossy transmission line (diffusive regime), the attenuation is due to ohmic losses represented in Fig. 1 by $R$. The characteristic impedance is no longer a pure real number and is given by $Z_{0}=1 / g \sqrt{R / 2 \omega C_{t o t} e^{-j \Pi / 4}}$ (Wong and Akinwande, 2011). Analyzing this expression, one can conclude that, for the metallic CNT, the voltage is delayed with respect to the current by $\Pi / 4$, presenting, thus, partially capacitive behavior. In addition, the amplitude of the signal becomes dependent upon the frequency $(\omega)$. The term $C_{\text {tot }}$ is the resulting capacitance of $C_{q}$ and $C_{e s}$ in series, according to the model presented in Fig. 1 , and $g$ is computed as $g=\sqrt{C_{t o t} / C_{q}}$.

The group velocity is given by $v_{g}=2 \sqrt{2 \omega / R C_{t o t}}$, which also depends on $\omega$. This dependence causes undesirable signal dispersion along the metal CNT. In order to reduce the effect of attenuation and scattering of the signal, one should keep both the length and the $C_{e s}$ of the metallic $\mathrm{CNT}$ as small as possible. To decrease the influence of $\omega$ in the $v_{g}$, the $C_{e s}$ must be much smaller than the $C_{q}$ (Wong and Akinwande, 2011).

In general, $\mathrm{CNT}$ as metallic interconnecting elements will have an advantage over the copper tracks when the CNT are in ballistic regime, as one can see in Fig. 2 (Wong and Akinwande, 2011; Javey and Kong, 2010).

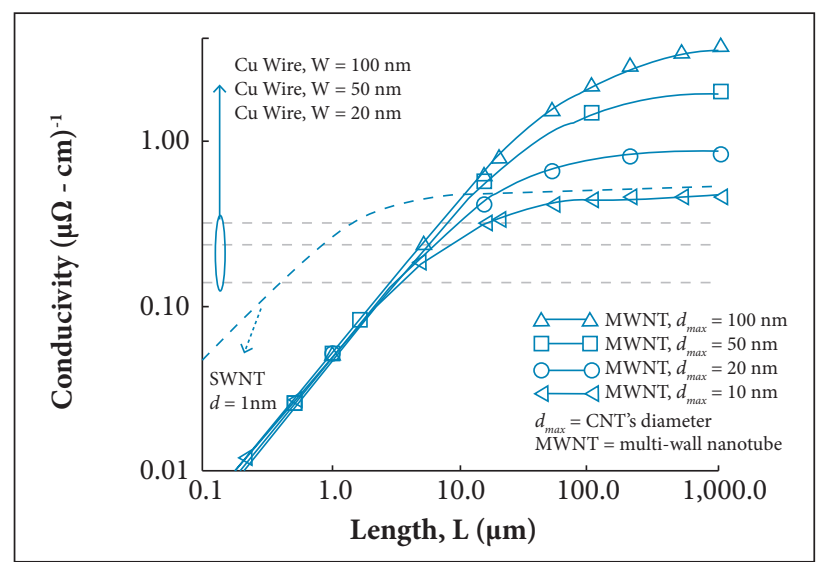

Figure 2. Conductivity for SWCNT and MWNT nanotubes as a function of length (Wong and Akinwande, 2011).

\section{NANODIODES WITH CNT}

In order to obtain rectifying $\mathrm{p}-\mathrm{n}$ junctions, one can use different methods in particular, doping impurities or intramolecular junctions. Intramolecular junctions in principle can be obtained by introducing defects in a CNT, using two different mechanisms. Firstly, by a mechanical deformation, which consists in bending of a CNT with respect to its axis. At the spot of deformation, some defects are introduced, which, in this case, are pentagonheptagon pairs, so that, in one side of the bending, there is a metallic CNT and, in the other side, a semiconductor CNT (Fig. 3a). Secondly, one can use a junction of two CNT with different diameters. One of the CNT behaves as a metal and the other one, as a semiconductor. At the point of junction, pentagon-heptagon pairs in hexagonal structure of the $\mathrm{CNT}$ are also introduced. Figure $3 \mathrm{~b}$ shows details of the junction (Yao et al., 1999; D’yachkov, 2006). 
According to Yao et al. (1999), the carbon nanotube shown in Fig. 4a is placed directly on three electrodes. The electrodes are over a film of silicon dioxide $\left(\mathrm{SiO}_{2}\right)$, which, in turn, is on a silicon (Si) wafer that has the function of a gate. At room temperature, the resistance of the carbon nanotube's upper segment between the two electrodes is $110 \mathrm{~K} \Omega$, shown in detail in Fig. 5a. This thread has metallic behavior and is independent of the applied gate voltage. On the other hand, the current-voltage characteristic of the carbon nanotube bend, also shown in Fig. 5a, has a highly non-linear and asymmetric behavior, resembling the currentvoltage characteristics of a diode rectifier. We can observe a very high increase in the current when applying a positive voltage on the top electrode when the voltage exceeds the threshold voltage, which is about $2 \mathrm{~V}$ (Fig. 5a). There is a very small increase in current for the reverse bias. This junction has an extremely high impedance for the voltages around $0 \mathrm{~V}$.

Figure $5 \mathrm{~b}$ represents current-voltage characteristics of metal-semiconductor junction in Fig. 4a. The electrodes are made of gold ( $\mathrm{Au}$ ) and titanium $(\mathrm{Ti})$ and have dimensions of $250 \mathrm{~nm}$ wide, $20 \mathrm{~nm}$ in thickness and less than $1 \mathrm{~nm}$ in height, which minimizes deformation of the CNT. The curve in Fig. 5a is obtained with the grounded gate. In Fig. 5b, the gate voltage from left to right ranges from $-4 \mathrm{~V}$ to $+2 \mathrm{~V}$.

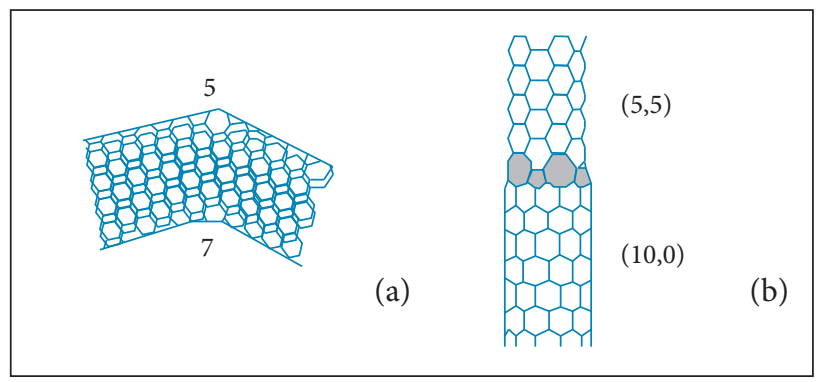

Figure 3. Examples of rectifying p-n junction (D’yachkov, 2006).

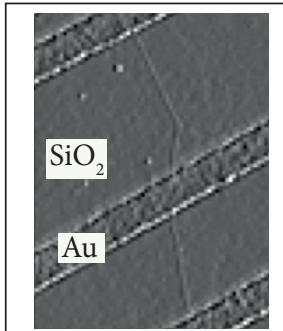

(a)

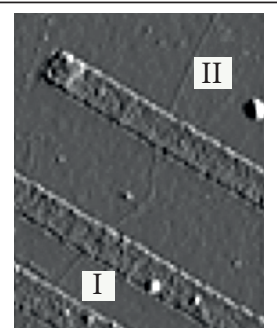

(b)



(c)
Figure 4. Sample image junction obtained by atomic force microscope (Yao et al., 1999): (a) CNT placed directly on 3 electrodes, (b) CNT folded that presents the effect of rectification, (c) the detail of the fold, in which, on one side of the fold, the CNT is metallic and, on the other one, it is semiconductor.

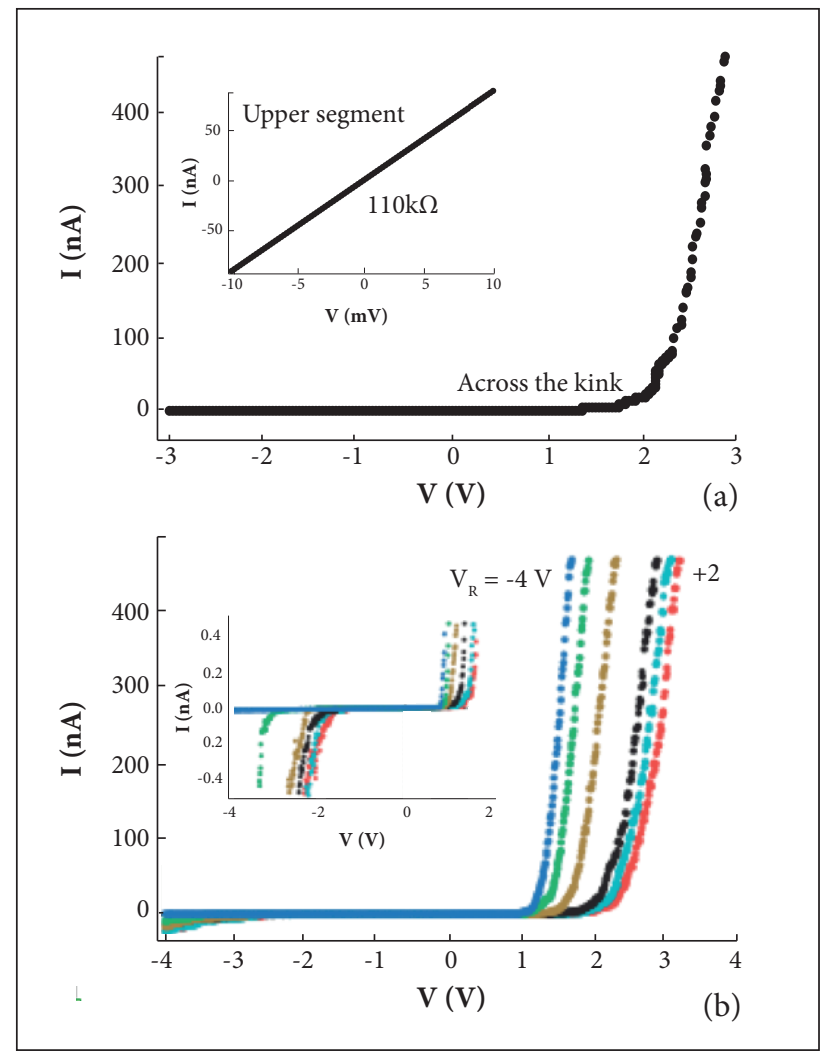

Figure 5. Current-voltage characteristic of metal-semiconductor junction in Fig. 4a.

The Schottky diode is another type of diodes of particular interest for applications in the range of $30 \mathrm{GHz}$ to $3 \mathrm{THz}$. The diodes that operate in this range are commonly used as detectors, mixers and frequency multipliers in the submillimeter wave region at room temperatures. The CNT diodes are characterized by their high switching speed and inherent suitability for applications at low voltages and high currents (Manohara et al., 2005; Huang et al., 2010).

In devices based on microelectronics in frequencies above a few $\mathrm{GHz}$, there are the inherent parasitic capacitance and resistance. The capacitance is proportional to the area of the junction and the resistance is inversely proportional to the mobility of the electrons. These undesirable capacitances and resistances are mainly due to the limitations of the manufacturing process of the device, as well as to the properties of the materials. These drawbacks severely limit the sensitivity of signal detection and signal generation in $\mathrm{THz}$ range. Typical values of signal power are of the order of a few microwatts for frequencies up to $1.5 \mathrm{THz}$ (Manohara et al., 2005).

From the standpoint of new materials, the CNT offer an excellent alternative in the design of high-frequency devices 
in $\mathrm{THz}$ range because of small areas of the junctions, which usually have a maximum of $2 \mathrm{~nm}$ in diameter. This leads to low capacitances of the order of tens $\alpha F / \mu m$. Besides, the CNT possess high electron mobility, reaching up to $200.000 \mathrm{~cm}^{2} /$ V.s (Manohara et al., 2005).

Unlike planar microelectronic Schottky diode junctions, the Fermi contact level does not control the properties of devices with CNT. As a result, the best threshold voltage for optimal performance of the device can be chosen (Manohara et al., 2005; Huang et al., 2010).

Figure 6 shows the basic schematic representation of a Schottky diode, whose design employs two different metals at the ends of the p-type CNT. The utilized metals have different work functions $(\Phi)$; one of them has a lower work function than the CNT in order to have the Schottky contact; the other one has a higher work function than the CNT in order to obtain the ohmic contact (Manohara et al., 2005).

In Fig. 6a, the Schottky contact is obtained with the use of Ti, and $\Phi_{T i}=4.33 \mathrm{eV}<\Phi_{N T C}$, with $\Phi_{N T C} \approx 4.9 \mathrm{eV}$. The ohmic contact is obtained with the use of platinum ( $\mathrm{Pt}$ ) possessing, and $\Phi_{P t}=5.65 \mathrm{eV}>\Phi_{N T C}$ (Manohara et al., 2005). There is a layer of $\mathrm{SiO}_{2}$ between the $\mathrm{Si}$ substrate and the CNT of approximately $400 \mathrm{~nm}$ of thickness.

Figure 7 shows the direct (DC) current-voltage characteristics of four different types of Schottky diode manufactured on the

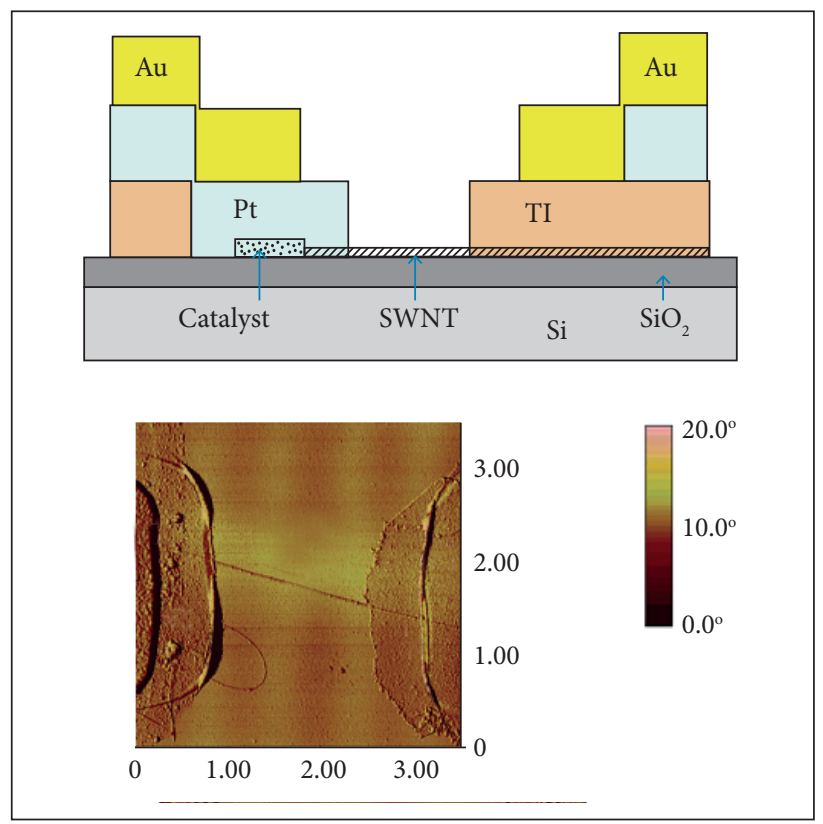

Figure 6. (a) Schottky diode's schematic representation, (b) typical image obtained by atomic force microscope (Manohara et al., 2005). same substrate and in the same batch with the zero gate voltage. All these diodes contain a single CNT with semi-conductive characteristic between the two contacts of different metals formed by Ti and Pt (Manohara et al., 2005). We can see in Fig. 7 that the current-voltage characteristics for the four diodes are quite different. These differences can be attributed to the characteristics of the CNT, such as, for example, its overall length and the overlap that it makes with the metallic contacts (Yang et al., 2005).

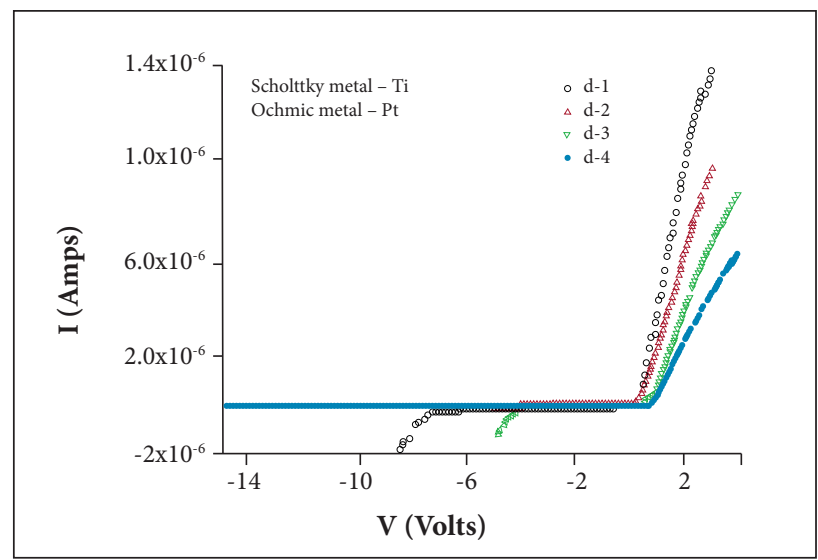

Figure 7. Current-voltage characteristics of four Schottky diodes (Manohara et al., 2005).

There is another type of diode, also for high frequency, which uses multiple CNT in parallel between the metal contacts of the device (Manohara et al., 2005). Each device has from 8 to 10 CNT. These nanotubes may have either metallic or a semiconductor behavior and this property is difficult to control. The presence of only one carbon nanotube with metal characteristics destroys the desired effect of rectification. Therefore, the CNT with metallic behavior were literally burned by a high current in the technological process that is described in Collins et al. (2001) so that all the remained tubes had semi-conductor properties. Figure 8 shows the change in current-voltage characteristic before and after the selective burning of metallic $\mathrm{CNT}$. The inset chart in this figure demonstrates the curve for the rectifier for low bias voltages.

Figure 9 shows the current-voltage curves of symmetric devices, i.e. those whose metallic contacts are made of the same material. All electrical measurements were made at room temperature in the air. In Fig. 9a, palladium (Pd) was used as contact metal, resulting in a behavior of a p-type semi-conductor with on-off current ratio of $10^{6}$. In Fig. 9a, it is shown in detail the image that was obtained through a scanning electronic microscope. In Fig. 9b, Ti was used as the metal contact, resulting 
in an ambivalent behavior, i.e. for negative bias voltages, the device features a semi-conductor p-type behavior and, for positive bias voltages, the same device shows a semi-conductor n-type behavior. The on-off ratio is about $10^{4}$. In Fig. 9c, aluminum (Al) was used as the metal contact, resulting in an on-off ratio of $10^{3}$ for negative gate bias voltages and an on-off ratio of $10^{6}$ for positive bias voltages.

As seen in Fig. 9, the simple change of the type of material used as electrical contact modifies the current-voltage characteristic of the device, and it can present both a p-type semi-conductor behavior and an n-type semi-conductor behavior or even ambivalent characteristics.

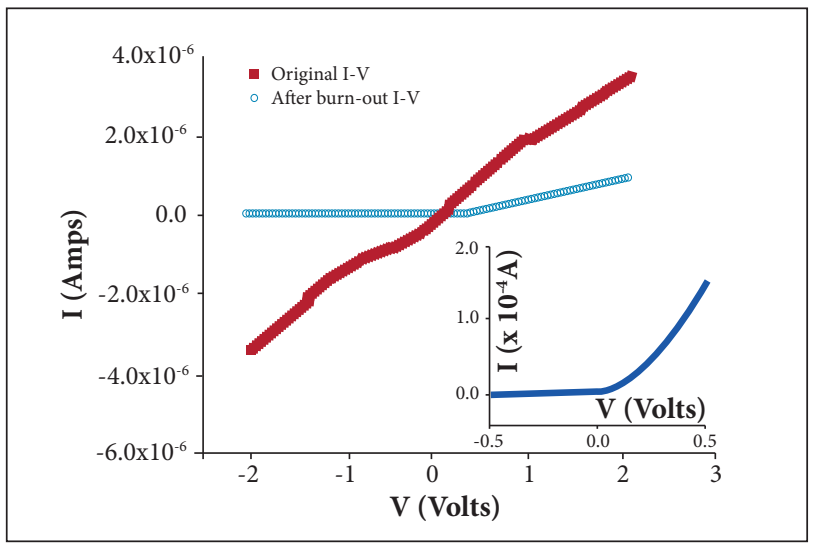

Figure 8. Current-voltage curve of Schottky diode with multiple CNT (Manohara et al., 2005).

\section{NANOTRANSISTORS BASED ON CNT}

In microelectronics, the basic structure of a field-effect transistor (FET) of solid state is composed of two metal electrodes called source and drain, which are connected by a channel in a semi-conductor. There is a third electrode, called the gate, which is isolated from the channel by a thin layer of $\mathrm{SiO}_{2}$. By varying the channel width, the gate bias voltage controls the current flow between the source and drain terminals.

In FET with CNT (CNFET), the Si semi-conductor channel is replaced by a SWCNT, which is inserted over the metal electrodes with high work function $\Phi>5 \mathrm{eV}$. These electrodes are typically made of Au or Pt (Avouris, 2002).

Back in 1998, there were the first demonstrations that CNT could be used in the design of FET (Adel and Smith, 2007). An example of this transistor is shown in Fig. 10. In this picture, a carbon nanotube is positioned at the top, interconnecting thus the two electrodes of noble metals. These electrodes are the source and drain terminals of the FET, which were fabricated



Figure 9. Current-voltage curves of a symmetric Schottky diode with three different metals as contact (Avouris, 2002).

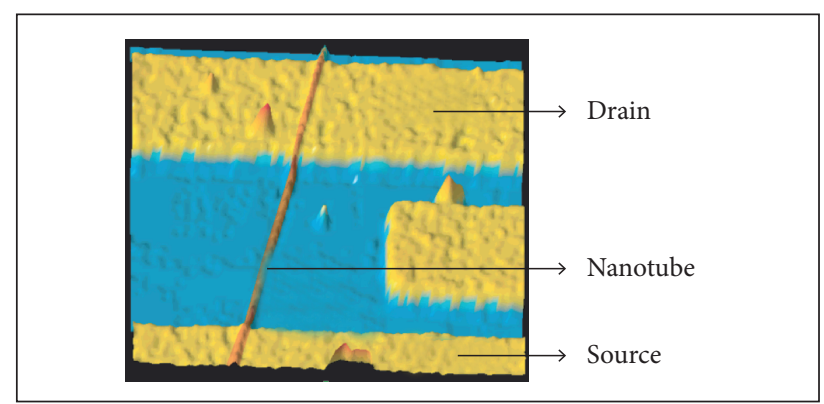

Figure 10. Image of field-effect transistor with CNT obtained by atomic force microscope (Adel and Smith, 2007).

at the top of $\mathrm{S} \mathrm{SiO}_{2}$ film, which, in turn, was deposited on a $\mathrm{Si}$ wafer. This Si wafer operates as a third terminal, which has the function of gate. These devices have the properties of a p-type 
FET with the ratio of on-off current of the order of $10^{5}$. They are characterized by a high parasitic resistance of contact, which is generally around $1 \mathrm{M} \Omega$, a low current between source and drain and a low transconductance of the order of $10^{-9} \mathrm{~A} / \mathrm{V}$.

In order to reduce the contact resistance and improve the features of the CNFET, the scheme shown in Fig. 10 can be modified as proposed in Adel and Smith (2007). In this assembly, there is an oxidized $\mathrm{Si}$ wafer placed on the $\mathrm{SiO}_{2}$, in which the SWCNT is dispersed. The source and drain terminals are fabricated on top of a SWCNT semi-conductor, according to the scheme in Fig. 11. The terminals are made of Ti or cobalt (Co). The curve of Fig. 12 was obtained at room temperature, with the drain, source and gate terminals made of Ti. The thickness of the $\mathrm{SiO}_{2}$ under the gate is $15 \mathrm{~nm}$. A reduction of the parasitic resistance is obtained by heat treatment between the metal and the SWCNT. As a result, the resistance was reduced from $1 \mathrm{M} \Omega$ to $30 \mathrm{~K} \Omega$, approximately. Figure 12 shows the output characteristics of this CNFET Adel and Smith (2007). The device in Fig. 11 can also be switched using a Si wafer as a back-gate.

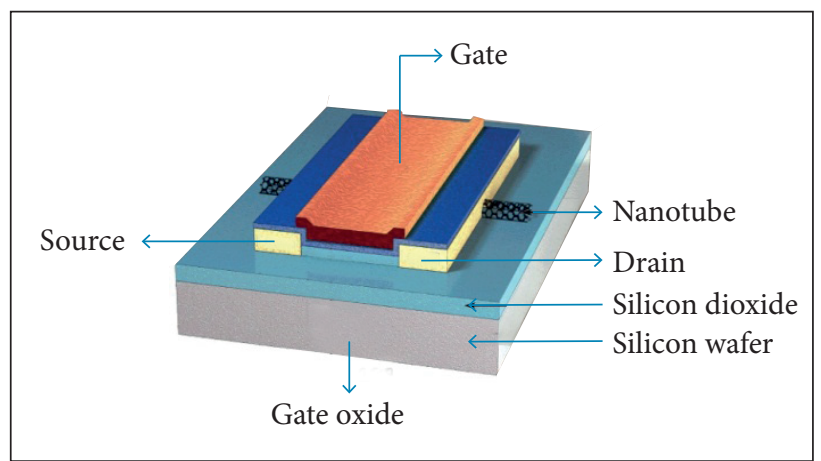

Figure 11. Schematic representation of field-effect transistor with CNT with top-gate (Adel and Smith, 2007).

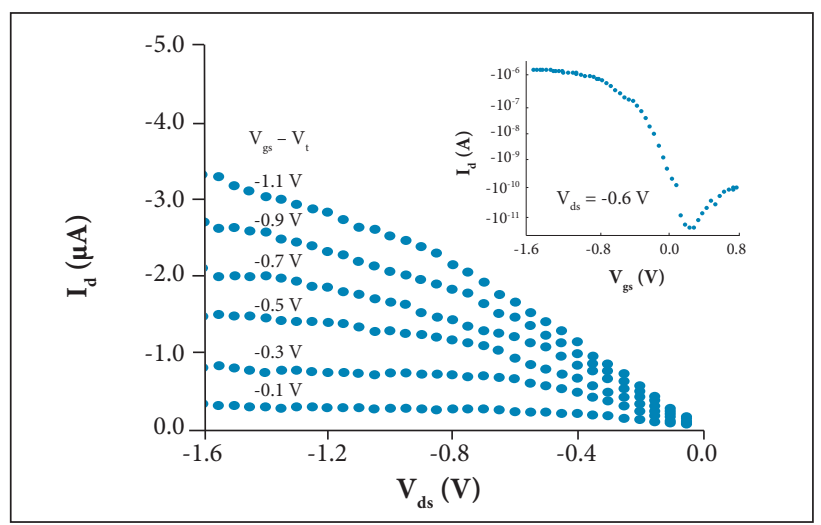

Figure 12. Electrical output characteristics of top-gate fieldeffect transistor with CNT with source, drain and gate terminals of titanium at room temperature (Adel and Smith, 2007).
Currently, there are several geometric configurations that can be used in implementation of the CNFET. The most commonly used are shown in Fig. 13 (Wong and Akinwande, 2011).

In the settings of Fig. 13, all of them retain some features in common with each other, such as the use of intrinsic CNT semi-conductors with the channel function, the use of metal contacts to the terminals of the source, drain and gate. The gate can be both top-gate and back-gate and, as a common use, all configurations make use of a dielectric or an oxide in order to isolate the gate terminal of the CNT.

The dynamics of CNFET operation is simple to understand. When a bias voltage is applied at the gate terminal of the CNFET, a perpendicular electric field appears in the channel of this device. This electric field controls the amount of charge carriers in the channel. The control can be fulfilled in two different ways: by top-gate, when the gate terminal is placed over the CNT, or by back-gate, when the gate terminal is underneath the Si wafer, which is heavily doped.

The top-gate controls the channel that is right below the gate terminal and the back-gate does the same control, but

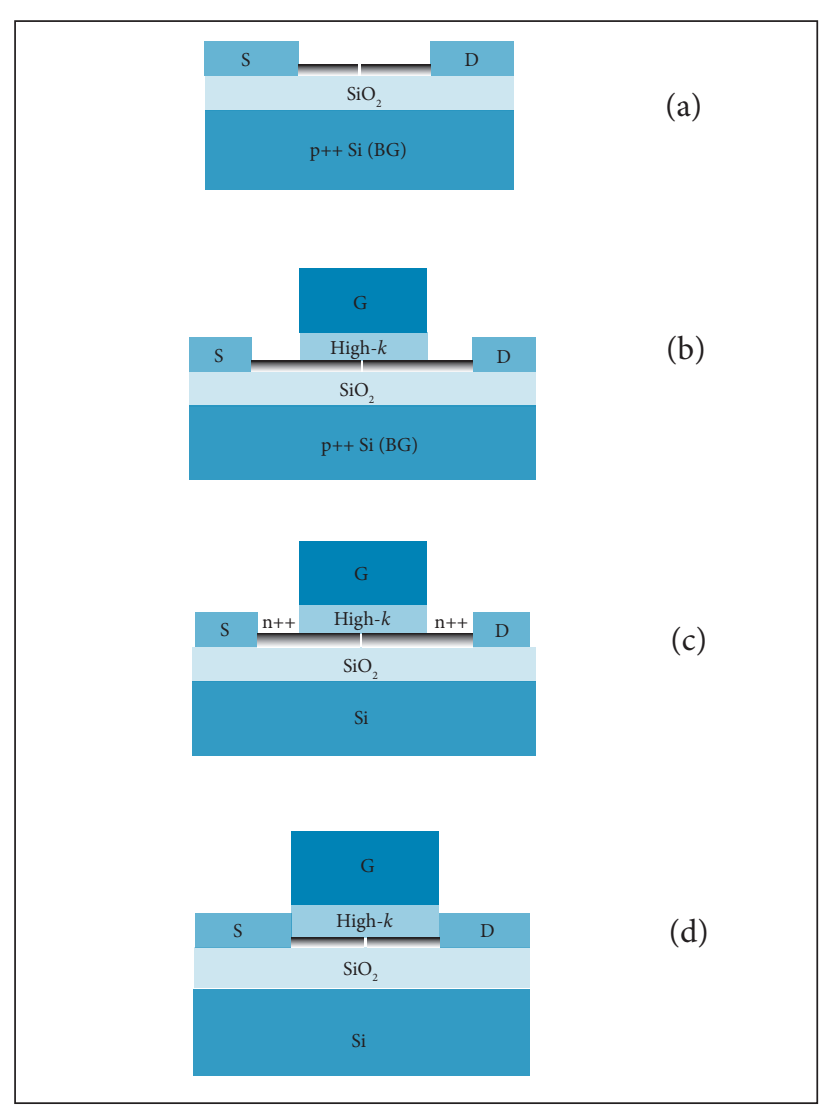

Figure 13. Geometric configurations commonly used in fieldeffect transistor with CNT (Wong and Akinwande, 2011). 
now, through an electrostatic doping in a region of the CNT near of the drain and source terminals in order to decrease the contact resistance between these terminals. This doping is done by electrostatic application of vertical electric field that induces electrons or holes near the region of the drain source terminals. Regardless of the type of control used, whether by a top-gate or a back-gate, there is an electric field due to the applied bias between drain and source. This electric field is responsible for the displacement of charge carriers between the drain and source terminals, whose intensity is controlled by the gate terminal.

Four basic geometry settings of CNFET are shown in Fig. 13. Figure 13a depicts the scheme of the device of Fig. 10, in which the Si substrate is heavily doped and plays the role of back-gate. The difference between Fig. 13b and Fig. $13 \mathrm{a}$ is that there is an additional electrode $\mathrm{G}$ which plays the role of top-gate. The top-gate is separated from the CNT by a high-k dielectric (the high-k dielectric has the dielectric constant $\mathrm{k}$ several times much greater than that of $\mathrm{SiO}_{2}$ ). Therefore, in this geometry, one can control the current flow between source and drain terminals, both through the top-gate and by the back-gate. Figure $13 \mathrm{c}$ uses the chemical doping in the regions close to the source and drain terminals, and Fig. 13d depicts the geometry of a top-gate with a selfaligned CNFET, which implies a perfect alignment, i.e. no space between the gate, source and drain metal contacts. Controlled devices through the top-gate are preferred when a greater control of the channel is desired, as well as the topgate allows the control of individual gates on a device with multiple transistors. Notice that the charge carriers in CNFET can be both electrons and holes (Wong and Akinwande, 2011).

In Table 1, the four charge transport schemes for CNFET are represented. These schemes are independent on the geometric setting. They are defined by the length, the mean free path of the CNT and the type of contact between the terminals, i.e. the ohmic or Schottky barrier. In Table $1, L$ is the length and $L_{m}$ is the mean free path of the CNT.

In the projects of CNFET devices, one needs to optimize the current between source and drain terminals. In this sense,

Table 1. The four transport regime of field-effect transistor with CNT (Wong and Akinwande, 2011).

\begin{tabular}{|c|l|l|}
\hline $\begin{array}{c}\text { Type of contact } \\
\text { between metal-CNT }\end{array}$ & $L<I_{m}$ & $L>I_{m}$ \\
\hline Ohmic & Ballistic & Diffusive \\
\hline Schottky barrier & Ballistic & Diffusive \\
\hline
\end{tabular}

projects with transparent contacts (i.e. ohmic contacts) and ballistic transport offer the best performance.

\section{DIFFERENCES BETWEEN 2-D METAL-OXIDE- SEMICONDUCTOR FIELD-EFFECT TRANSISTOR AND 1-D CNFET}

In Table 2, a comparison between the 2-D metal-oxidesemiconductor field-effect transistor (MOSFET) and 1-D CNFET is given. Due to the 1-D nature of CNT, in the linear region, the electrons can move only in one direction, i.e. either forward or backward and the charge transport can be ballistic. As for the MOSFET, the electrons can move in the plane and the electrons transport is diffusive. In the active regions of the MOSFET, the current of saturation is due to the pinch-off that occurs in the channel and, as a consequence, the drift rate no longer depends on the drain voltage (Martel et al., 2001). As for CNFET, the current of saturation occurs due to disappearance of the charge carriers from the drain terminal. This implies that the current does not depend on the drain voltage, unlike of what occurs with the MOSFET (Wong and Akinwande, 2011).

Table 2 shows explicitly that the device current in the on state $\left(I_{O N}\right)$ for the MOSFET is controlled mainly by the drift velocity $\left(u_{e f f}\right)$, which depends on the properties of the material. Whereas for CNFET this current mainly depends on the physical properties of states of the electrons in the solid, such as the band-gap structure $\left(E_{g}\right)$, the Boltzmann constant $\left(k_{s}\right)$ depends on the temperature $(T)$ and the quantum resistance $\left(R_{q}\right)$ (Wong and Akinwande, 2011). The parameter $u_{\text {eff }}$ is the drift velocity, which is an indicative of the average scattering that the loads suffer in the solid; $L$ and $W$ are the length and width of the channel, respectively; $C_{o x}$ is the capacitance of the insulating placed between the top-gate and the channel; $\varphi_{s}$ is

Table 2. Difference between 2-D MOSFET and 1-D CNFET (Wong and Akinwande, 2011).

\begin{tabular}{|c|c|c|c|}
\hline \multicolumn{2}{|c|}{$\begin{array}{c}\text { 2-D MOSFET } \\
I_{\text {oN }} \approx u_{\text {eff }} \frac{C_{o x} W}{2 L}\left(V_{G}-V_{T}\right)^{2}\end{array}$} & \multicolumn{2}{|c|}{$I_{O N} \approx \frac{k_{B} T}{e R q} \ln \left(1+e^{\frac{2 e \varphi_{s}-E_{g}}{2 k_{B} T}}\right)$} \\
\hline Parameter & Origins & Parameter & Origins \\
\hline (i) $u_{\text {eff }}$ & Drift velocity & (i) $\varphi_{s}$ & Gate coupling \\
\hline (ii) $I / L$ & Drift velocity & (ii) $E_{g}$ & Band structure \\
\hline (iii) $C_{o x} / W$ & Charge sheet & (iii) $R_{q}$ & $\begin{array}{l}\text { Quantum } \\
\text { transport }\end{array}$ \\
\hline (iv) $V^{2}$ & $\begin{array}{l}\text { Drift velocity } \\
\text { and charge } \\
\text { sheet }\end{array}$ & (iv) $k_{g} T$ & $\begin{array}{l}\text { Fermi-Dirac } \\
\text { distribution }\end{array}$ \\
\hline
\end{tabular}


the electrostatic surface potential; $V_{G}$ is the gate voltage and $V_{T}$ is the threshold voltage. The other parameters of Table 2 have already been described in the text.

A more detail information about the topics of this paper can be found in references below.

\section{CNT DEVICE SIMULATIONS}

In the nanoscale simulations, one needs to consider the effects of a single atom on the characteristics of the device. In the atomic scale, the effects of the individual vacancies and impurities start to have a real influence in the performance of the device.

There are two main steps in the nanoelectronic studies. The first one is a solution of the electronic structure problem that deals with the behavior of electrons' band energy inside of the material as well the DOS in such energy levels. In practice, this study gives us the information about the band gap and the energy levels where the device will work properly. Two categories of methods are used in practice: $a b$ initio approaches to solve the Schrödinger equation based on first principles. An example of such methods is density-functional theory (DFT). The second category is semi-empirical methods where the electronic structure is calculated by using adjustable parameters obtained, for example, from experiments.

The second step is the study of electronic transport, i.e. the behavior of the electronic current inside the material. The problem here is usually to obtain the I-V (current-voltage characteristic) curves. In this step, it is necessary to consider the electrons as wave functions instead of particles. That is, it is important to take into account the quantum effects that appear in the atomic scales. At these scales, the channel of the device (the active region of interest) is frequently shorter than the mean-free path of the material. Thus, one has to take into account the potential profile of the central region; has to consider quantum mechanical equations and approximation, such as Schrödinger equation; needs to apply, in the right way, the boundary conditions; and has to consider the nonequilibrium situation because the transmission property of the electron is itself bias dependent - that is the problem is nonlinear and, for each value of applied voltage, one has a different transmission curve. A combination of the Landauer approach with the non-equilibrium Green function (NEGF) method is now widely used in the analysis and design of nanoscale devices. Quantum transport in nanotubes and other nanoelectronic devices is described in more details in (Wong and Akinwande, 2011; Javey and Kong, 2010; Stokbro et al., 2010; Blom and Stokbro, 2011; Datta, 2005; Nazarov and Blanter, 2009).

\section{CONCLUSION}

Some special electrical, optical and magnetic properties, the mechanical strength and chemical stability make the CNT one of the promising nanomaterials to be used in nanoelectronics.

Based on the available literature, this study shows that the devices with CNT are very promising for replacing microelectronic devices that employ $\mathrm{Si}$, germanium $(\mathrm{Ge})$ or GaAs as semi-conductor elements. The use of CNT as a metal to interconnect elements in order to replace the copper tracks is advantageous when the mean free path is smaller than the link track.

The use of CNT in the projects of diodes and FETs allows one to increase the frequency range of these devices, sometimes even to THz. Possible applications of such devices are detectors, mixers, frequency multipliers, among others.

However, there are still some challenges to be overcome in order to raise the manufacturing process of these CNT devices to a commercial scale, such as control of the physical and electronic properties in the CNT growth process. The production of CNFET is still complicated, expensive and requires a lot of time.

\section{REFERENCES}

Adel, S. and Smith, K.C., 2007, "Microeletrônica", Fifth Ed., Prentice Hall, São Paulo, Brazil.

Avouris, P., 2002, "Molecular Electronics with Carbon Nanotubes", Accounts of Chemical Research, Vol. 35, No. 12, pp. 1026-1034. doi: 10.1021/ar010152e
Blom, A. and Stokbro, K., 2011, "Towards Realistic Atomic-Scale Modeling of Nanosacale Devices", IEEE Conference on Nanotechnology, pp. 1487-1492. doi: 10.1109/NANO.2011.6144584

Collins, P.G., Arnold, M.S. and Avouris, P., 2001, "Engineering Carbon Nanotubes and Nanotube Circuits Using Electrical Breakdown", Science, Vol. 292, No. 5517, pp. 706-709. doi: 10.1126/science.1058782 
D’yachkov, P.N., 2006, "Carbon Nanotubes: Structure, Properties and Applications", BINOM, Moscow, Russia.

Datta, S., 2005, "Quantum transport. Atom to transistor", Cambridge University Press, Cambridge, UK. 404p.

Dragoman, M. and Dragoman, D., 2006, "Nanoelectronics: Principles and Devices", Artech House, Boston, USA/London, UK. 426p.

Huang, L., Chor, E.F., Wu, Y. and Guo, Z., 2010, "Fabrication of Single-Walled Carbon Nanotube Schottky Diode with Gold Contacts Modified by Asymmetric Thiolate Molecules", Carbon, Vol. 48, No. 4, pp. 1298-1304. doi:10.1016/j.carbon.2009.12.003

lijima, S., 1991, "Helical Microtubules of Graphitic Carbon", Nature, Vol. 354, pp. 56-58,. doi: 10.1038/354056aO

Javey, A. and Kong, J., 2010, "Carbon Nanotube Electronics", Springer, New York, USA.

John, D.L., Castro, L.C. and Pulfrey, D.L., 2004, "Quantum Capacitance in Nanoscale Device Modeling", Journal of Applied Physics, Vol. 96, No. 9, pp. 5180-5184.

Manohara, H.M., Wong, E.W., Schlecht, E., Hunt, B.D. and Siegel, P.H., 2005, "Carbon Nanotube Schottky Diodes Using Ti-Schottky and Pt-Ohmic Contacts for High Frequency Applications", Nano Letters, Vol. 5, No. 7, pp. 1469-1474
Martel, R., Derycke, V., Lavoie, C., Appenzeller, J.and Chan, K.K., 2001, "Ambipolar Electrical Transport in Semiconducting Single-Wall Carbon Nanotubes", Physical Review Letters, Vol. 87, No. 25, pp. 256805. doi: 10.1103/PhysRevLett.87.256805

Nazarov, Y. and Blanter, Y., 2009, "Quantum Transport. Introduction to Nanoscience", Cambridge University Press, Cambridge, UK.

Sadiku, M.N.O., 2004, "Elementos de Eletromagnetismo", Third Ed., Bookman, Porto Alegre, Brazil.

Stokbro, K., Peterson, D., Smidstrup, S., Blom, A., Ipsen, M. and Kaasbjerg, K., 2010, "Semiempirical Model for Nanoscale Device Simulations", Physical Review B, Vol. 82, No. 7, 075420. doi: 10.1103/PhysRevB.82.075420

Wong, H.S.P. and Akinwande, D., 2011, "Carbon Nanotube and Graphene Device Physics", Cambrigde University Press, Cambrigde, UK. 262p.

Yang, M.H., Teo, K.B.K., Milne, W.I. and Hasko, D.G., 2005, "Carbon Nanotube Schottky Diode and Directionally Dependent Field-Effect Transistor Using Asymmetrical Contacts", Applied Physics Letters, Vol. 87, 253116.

Yao, Z., Postma, H.W.C., Balents, L., and Dekker, C., 1999, "Carbon Nanotube Intramolecular Junctions", Nature, Vol. 402, pp. 273-276. doi:10.1038/46241 